\title{
Perceptions communautaires du couple sécheresse/déboisement dans le sous-bassin versant de Koumagou à Boukoumbé (Nord-Ouest Bénin)
}

\author{
Ibouraima Yabi, Mahamoud N’tcha Tchanati, and Akindélé Akibou \\ Laboratoire Pierre PAGNEY Climat, Eau, Ecosystème et Développement (LACEEDE), Département de \\ Géographie et Aménagement du Territoire (DGAT), Faculté des Sciences Humaines et Sociales (FASHS), \\ Université d'Abomey-Calavi (UAC, Bénin), Bénin \\ Correspondence: Ibouraïma Yabi (yafid2@yahoo.fr)
}

Published: 16 November 2021

\begin{abstract}
Résumé. Au-delà des analyses climatologiques et statistiques, la présente communication s'intéresse aux connaissances communautaires relatives au couple sécheresse-déboisement qui sont les principaux moteurs de la dynamique environnementale dans le sous bassin versant de Koumagou au Nord-Ouest du Bénin. Sur la base des investigations auprès d'un échantillon de 180 personnes, les perceptions communautaires relatives aux manifestations, causes et conséquences des deux fléaux sans oublier les mesures d'adaptation, sont analysées. Des paramètres de la statistique descriptive ont été utilisés pour le traitement des données. La sécheresse est causée par le déboisement massif et la colère des dieux alors que l'agriculture et l'exploitation forestière sont perçues comme la cause du déboisement. Les ressources en eau, les ressources végétales et les sols sont les plus affectés selon les répondants avec des conséquences socioéconomiques. Les mesures d'adaptation concernent l'ajustement des pratiques culturales, le reboisement, la création des aires protégées communautaires, la mobilisation des eaux souterraines.
\end{abstract}

Abstract. Beyond climatological and statistical analyzes, this communication focuses on community knowledge relating to the combination of drought and deforestation, which are the main drivers of environmental dynamics in the Koumagou sub-watershed in north-west Benin. Based on investigations of a sample of 180 people, community perceptions relating to the manifestations, causes and consequences of the two scourges, not to mention adaptation measures, are analyzed. Descriptive statistics parameters were used for data processing. Drought is caused by massive deforestation and the wrath of the gods while agriculture and forestry are seen as the cause of deforestation. Water resources, plant resources and soils are the most affected according to the respondents with socio-economic consequences. The adaptation measures concern the adjustment of cultural practices, reforestation, the creation of community protected areas, the mobilization of groundwater.

\section{Introduction}

La dégradation inquiétante des ressources naturelles et ses corolaires sont sans conteste une des préoccupations majeures actuelles de l'humanité. En effet, au-delà de leur importance écologique, les ressources naturelles contribuent également à améliorer et à diversifier les activités socio- économiques, participent à la création d'emplois et constituent une source de recettes pour les État (Ndiaye et Ndiaye, 2013). Ainsi, en termes économiques, le capital naturel représente un quart de l'ensemble des richesses des pays à faible revenu, et elles sont souvent la principale source de revenu pour les populations les plus démunies (OCDE, 2009). C'est au regard de cette réalité que la gestion durable de l'en- 
vironnement et des ressources naturelles est essentielle à la réduction de la pauvreté dans les zones rurales des pays en développement (FIDA, 2011).

Malgré l'importance des ressources naturelles, elles font l'objet de pression aussi d'origine aussi bien naturelle qu'anthropique. Au nombre des facteurs naturels figure la sécheresse (OMM et GWP, 2016; Dardel, 2014). Or, les pays Ouest-Africains sont touchées par des graves sécheresses, notamment au cours des décennies 1970 et 1980 et la tendance n'est pas encore totalement normalisée par l'amélioration des cumuls pluviométriques à partir des années 1990. Ces sécheresses qui ont particulièrement touché les Pays pays sahéliens n'ont pas épargné les pays côtiers comme tel que le Bénin considéré comme plus humides. et Elles ont eu des conséquences sur les composantes écologiques, le couvert végétal en particulier. Les facteurs humains de dégradation des ressources naturelles quant à eux, concernent, l'agriculture, le pastoralisme, l'urbanisation, l'exploitation du bois, etc. En effet, une utilisation des terres au détriment des écosystèmes naturels a marqué les trois dernières décennies pour des motivations diverses : exploitation minière, étalement urbain, expansion agricole à travers le monde (Ndiaye et Ndiaye, 2013).

Le Bénin en général et le sous bassin de Koumagou en particulier (Fig. 1) n'est pas en marge de cette tendance à la dégradation des ressources naturelles en lien avec les causes naturelles (la sécheresse notamment) et les activités humaines.

La présente recherche s'intéresse particulièrement aux perceptions communautaires relatives aux effets du couple sécheresse/déboisement dans ce sous bassin à Boukoumbé. En effet, la connaissance et la prise en compte des perceptions, connaissances ou logiques communautaires sont capitales dans le processus de planification des mesures de gestion durable des ressources naturelles (Wicander, 2016) de cette entité hydro-écologique.

\section{Données et méthodes}

Les informations utilisées sont relatives aux perceptions ou connaissances des populations sur la sécheresse (ampleur et fréquence) et le déboisement. Il s'agit d'identifier, selon les populations locales, les causes et conséquences de ces phénomènes sur les composantes environnementales (eaux, végétation et sol). La taille minimale ( $n$ ) de l'échantillon est déterminée par la formule de Dagnélie utilisée par Akouehou et al. (2014) :

$n=\frac{U_{1-\frac{\alpha}{2}}^{2} \times(1-P)}{d^{2}}$

Dans cette équation, $n=$ taille minimale de l'échantillon considérée; $P=$ proportion des personnes capables de fournir des informations $; U_{1-\alpha / 2}=$ valeur de la loi normale à la valeur de probabilité $1-\alpha / 2$ (est de 1.96 avec $\alpha=5 \%$ ); $d$ : marge d'erreur de l'estimation fixée à une valeur de $6 \%$. La valeur de $P$ a été estimée à 0.8 au terme d'une enquête exploratoire qui a permis de sonder 60 personnes choisies au hasard. Sur la base de ces valeurs, l'application numérique de formule permet d'obtenir $n=177$, arrondie à 180 . Cet échantillon est réparti de façon proportionnelle dans les 4 arrondissements concernés par le sous bassin (Korontière : 36; Tabota : 53; Natta : 44; et Manta : 47). Le facteur genre est également pris en compte dans le choix des personnes. Ainsi, les personnes d'âges compris entre 25 et 55 ans représentent $65 \%$, contre $35 \%$ pour les plus de 55 ans. Les hommes représentent $78 \%$ des enquêtés contre $22 \%$ pour les femmes.

Un questionnaire a permis de faire des entretiens individuels. Des possibilités sont également offertes aux répondants de faire des commentaires libres sur les aspects abordés. En outre, des séances d'observations individuelles et directes ont été organisées pour mieux apprécier les formes de déboisement et les problèmes de dégradation qui en découlent.

Les informations collectées ont été codifiées manuellement et les questionnaires ont été dépouillés dans une base de données. Le traitement des informations ethnoenvironnementales collectées a nécessité l'utilisation des paramètres de la statistique descriptive (fréquence, illustrations graphiques).

\section{Résultats}

\subsection{Perceptions locales des causes de la sécheresse et du déboisement}

Les résultats indiquent que les populations du bassin perçoivent une augmentation de la fréquence $(85 \%)$ et de l'ampleur de la sécheresse (65\%). Le déboisement est devenu massif selon $91 \%$ des répondants. Ils ont indexé aussi bien les actions humaines que les facteurs naturels comme cause de cette tendance inquiétante (Fig. 2).

La Fig. 2 montre que la sécheresse est principalement causée par le déboisement $(78 \%)$, et la colère des dieux $(62 \%)$ alors que l'agriculture $(83 \%)$ et l'exploitations forestières (64\%) sont majoritairement perçues comme les causes du déboisement. Outre ces causes citées par la majorité, d'autres mobiles comme la variabilité naturelle du climat (sécheresse), les feux de végétation (déboisement), la forte chaleur (sécheresse) sont évoqués mais de façon minoritaire.

Pour expliquer la sécheresse, la variabilité climatique et le déboisement sont plus cités par les jeunes (ayant accès aux informations) alors que la colère divine est plus indexée par les vieux plus attachée à la tradition. Mais, les 2 catégories d'acteurs ont tous perçu l'agriculture et l'exploitation forestière (bois de chauffe, charbon de bois et bois d'œuvre) comme étant les principales causes des 2 fléaux qui sévissent dans le milieu.

Dans l'ensemble, les perceptions ont plus ciblé les causes liée directement ou non aux activités humaines. Ces résultats sont semblables aux conclusion de Hosonuma et al. (2012) et 


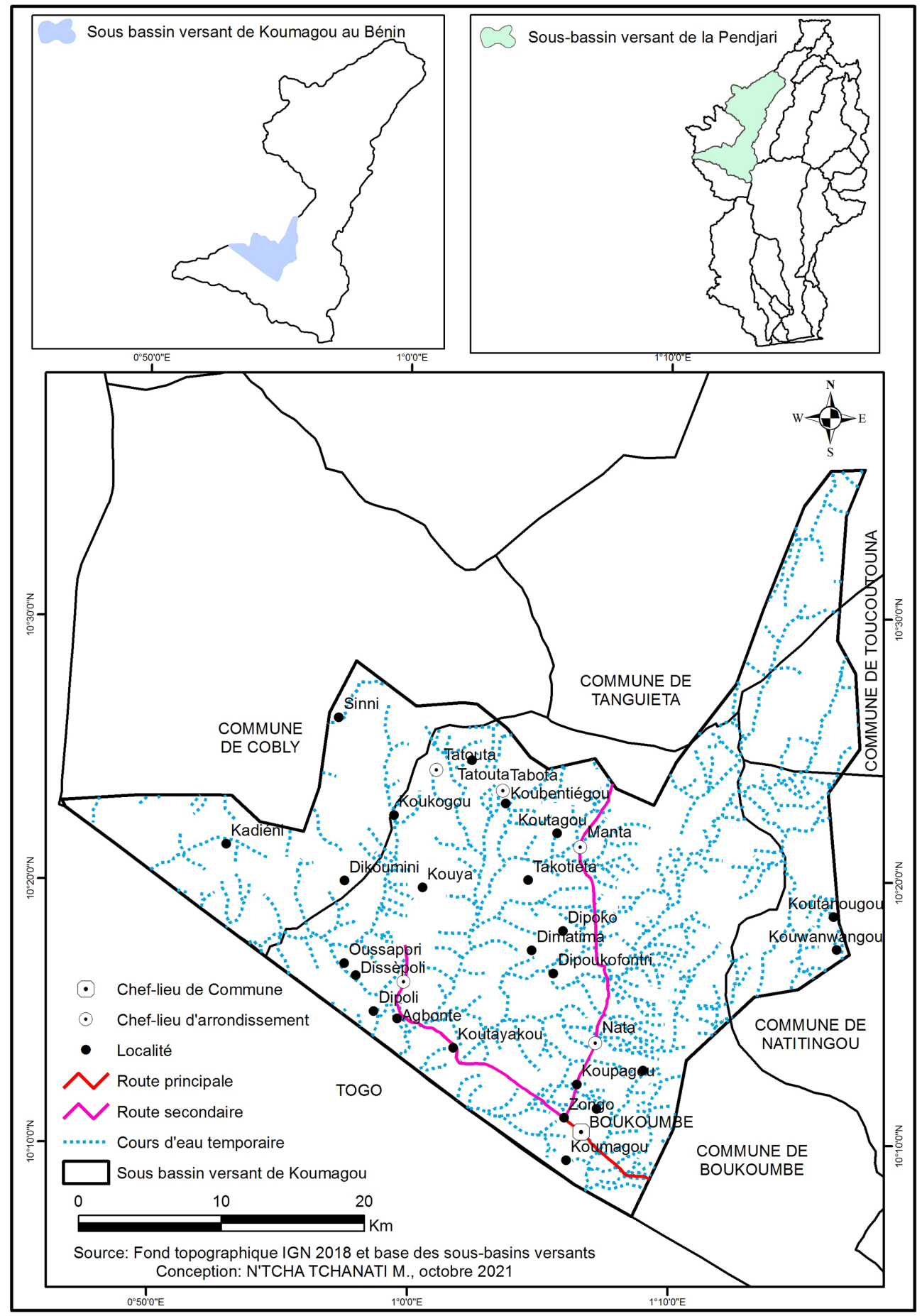

Figure 1. Situation géographique du sous-bassin de Koumagou à Boukoumbé.

de Gilet et al. (2016) qui ont mis en évidence l'agriculture extensive, l'exploitation du bois et l'extraction minière comme principaux facteurs directs de la dégradation du déboisement dans le monde. Au Bénin,

Arouan et al. (2016), Toko Imorou et al. (2019) ont obtenu des résultats similaires sur le rôle prégnant des actions hu- maines sur la dégradation du couvert végétal. Il en ressort que les perceptions paysannes sont globalement en phase avec les résultats d'analyses scientifiques. Il convient d'analyser les conséquences environnementales de ces phénomènes selon les perceptions. 


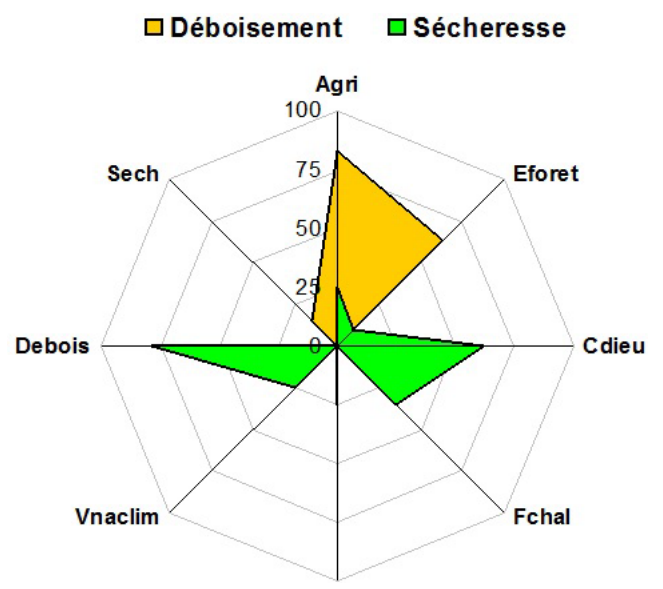

Figure 2. Causes du déboisement et de la sécheresse selon les populations locales. Légende : Agri=production agricole ; Apas = activité pastorale $;$ Eforet = exploitations forestières ; Cdieux $=$ Colère des dieux $;$ Fchal $=$ Forte chaleur $;$ Fvege $=$ feux de végétation; Vnaclim = variabilité naturelle du climat; Debois $=$ Déboisement $;$ Sech $=$ sécheresse.

\subsection{Effets perçus de la sécheresse et du déboisement}

Selon les répondants, les principales composantes environnementales, sources de moyens d'existence sont affectées par le déboisement et la sécheresse (Fig. 3). Ainsi, les ressources en eau (rivières, marigots, mares, etc.), sont devenues plus rares $(80 \%)$, disparaissent $(55 \%)$, tarissent tarissement précocement $(40 \%)$ et se comblent (35\%).

S'agissant des ressources forestières (espèces ligneuses, produits forestiers non ligneux), elles subissent des changements comme la raréfaction d'espèces (65\%), la disparition d'espèces $(45 \%)$, et la dégradation des écosystèmes caractéristiques $(55 \%)$.

Les sols quant à eux sont sujets à l'érosion (75\%), à la perte de fertilité ( $45 \%)$ et au compactage ( $25 \%)$. Dans l'ensemble, les femmes (ayant la charge de l'approvisionnement en eau du ménage et impliquées dans la production maraichères) ont plus cité la disparition et le tarissement précoce des sources d'eau alors que les agro-éleveurs ont plus perçu la dégradation des ressources forestières et la raréfaction/tarissement des ressources en eau. En effet, ils ont insisté sur le fait que la disponibilité de certaines espèces fourragères comme : Daniellia oliveri, Pterocarpus erinaceus, Afzelia africana, Khaya senegalensis, sont devenues rares. L'agriculteur quant à lui est plus sensible à la dégradation des sols qui sont le principal support de son activité. Ils pensent en effet que les sols sont plus dégradés et sont moins productifs, ce qui se traduit par la baisse des rendements agricoles.

Dans tous les cas, les activités et moyens d'existence des communautés sont touchées d'une manière ou d'une autre selon les enquêtes. Il s'en suit des retombées socioéconomiques négatives (pauvreté, instabilité du disponible alimentaire, tensions entre les acteurs, autour des ressources dis-

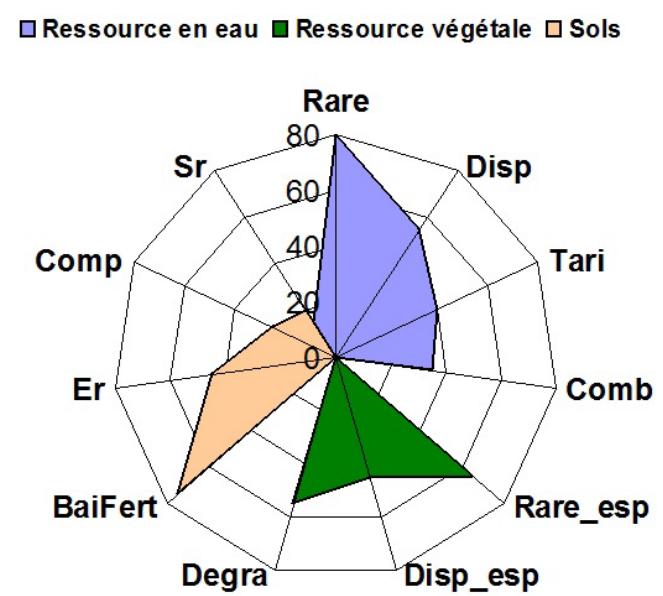

Figure 3. Effets du déboisement et de la sécheresse selon les perceptions. Légende : Rare $=$ raréfaction; Disp=disparition ; Tari $=$ tarissement $\quad$ précoce $; \quad$ Comb $=$ Comblement Rare_esp = raréfaction d'espèces ; Disp_esp=disparition d'espèces; Degra = dégradation d'écosystèmes; BaiFert = baisse de fertilité $; \mathrm{Er}=$ Erosion $;$ Comp $=$ Compactage $; \mathrm{Sr}=$ sans réponse.

ponibles). Le croisement des différentes perceptions permet de constater l'existence d'un cercle vicieux avec comme maillons : activités humaines, déboisement/sécheresse, dégradation des ressources naturelles, altération des moyens d'existence des communautés dans le sous bassin. Plusieurs auteurs comme : Claude (1989), Al Hamndou et RequierDesjardins (2008); Ndiaye et Ndiaye (2013), Ahononga et al. (2020), ont abouti aux conclusions identiques.

Pour tenter de limiter l'ampleur des effets néfastes, des mesures d'adaptation sont mises en œuvre par les paysans.

\subsection{Mesures communautaires d'adaptation et perspectives}

Les mesures d'adaptations citées concernent l'ajustement des pratiques culturales fondées sur les savoirs endogènes et les suggestions de l'encadrement rural. Il s'agit des pratiques agroforestières, des pratiques de Gestion Durable de Terre (GDT), la mise en valeur agricole de bas-fonds, etc. Il y a également des initiatives de reboisement qui permettent l'enrichissement des aires dégradées, des plantations agroforestières avec l'utilisation des plantes à valeur socioéconomique, etc. La création des aires protégés communautaires est également citée pour préserver les écosystèmes naturels sans oublier la mobilisation des eaux souterraines à travers les puits à vocation domestique et agricoles. A ces mesures, s'ajoutent l'organisation des prières et cérémonies en cas de sécheresse sévère qui menace la réussite de la campagne agricole. Ces mesures mobilisent plus les personnes âgées à faible niveau d'instruction.

Ces mesures visent à la fois à maintenir la couverture végétale, les services écosystémiques du milieu et l'améliora- 
tion des conditions de vie des populations. L'efficacité et la pertinence de ces mesures restent à évaluer. Elles témoignent toutefois de la proactivité des communautés à faire face aux défis environnementaux (Boko, 1988; Afouda et al., 2014; Yabi (2019). Il conviendrait de les analyser pour en ressortir celles qui sont durables et qui pourraient être vulgarisées. Les associations villageoises, les ONGs, les chefs traditionnels, les autorités locales devraient être associés à cet exercice dans le cadre d'une démarche participative et inclusive.

\section{Conclusions}

La présente recherche a permis de savoir que les communautés riveraines du sous bassin de Koumagou sont conscientes des dangers que constituent le déboisement et la sécheresse. Ces fléaux sont essentiellement causés par les activités humaines notamment l'agriculture et l'exploitation forestière selon les perceptions communautaires. Les principales composantes environnementales à savoir la végétation, les ressources en eau et les sols sont affectées par ces phénomènes d'après les répondants. Il s'ensuit un amenuisement de moyens d'existence des populations essentiellement tirés de l'agriculture. Face à l'ampleur de ces fléaux et de leurs effets, les populations mettent en œuvre des mesures d'adaptations qui concernent l'ajustement des pratiques culturales, les initiatives de reboisement, la création des aires protégées communautaires, la mobilisation des eaux souterraines.

La lutte contre la sécheresse et le déboisement passe par la mise en place des mesures participatives d'adaptation et de mitigation. Les bonnes pratiques communautaires actuelles pourraient être mises à contribution à cet effet.

Disponibilité des données. Acune donnée utilisée dans cet article n'est disponible au public.

Collaborateurs. IY a initié la recherche, a donné des orientations méthodologiques et a participé à la rédaction du manuscrit. MNT a participé à l'élaboration des outils de collecte des données, a participé aux enquêtes de terrain et a participé à la rédaction du manuscrit. AA a supervisé les enquêtes de terrain, a participé au traitement des données et a participé à la rédaction du manuscrit.

Intérêts concurrents. Les auteurs déclarent qu'ils n'ont aucun conflit d'intérêts.

Clause de non-responsabilité. Publisher's note : Copernicus Publications remains neutral with regard to jurisdictional claims in published maps and institutional affiliations.
Déclaration du numéro spécial. This article is part of the special issue "Hydrology of Large River Basins of Africa". It is a result of the 4th International Conference on the "Hydrology of the Great Rivers of Africa", Cotonou, Benin, 13-20 November 2021.

Remerciements. Les auteurs remercient sincèrement les évaluateurs qui ont contribué à l'amélioration du manuscrit et les organisateurs du colloque.

\section{Références}

Ahononga, F. C., Gouwakinnou, G. N., Biaou, S. S. H., et Biaou, S. : Vulnérabilité des terres des écosystèmes du domaine soudanien au Bénin de 1995 à 2015, Bois et Forêts des Tropiques, 346, 3550, 2020.

Afouda, F., Salako, M. P., et Yabi, I. : Instabilité intra-saisonnière des pluies de la grande saison agricole dans la commune de Kétou au Bénin, Revue de Géographie du Laboratoire Leïdi, 2, $26-$ 47, 2014.

Al Hamndou, D. et Requier-Desjardins, M. : Variabilité climatique, désertification et biodiversité en Afrique : s'adapter, une approche intégrée, VertigO [En ligne], 8, https://doi.org/10.4000/vertigo.5356, 2008.

Arouna, O., Eténé, C. G., et Issiako, D. : Dynamique de 1'occupation des terres et état de la flore et de la végétation dans le bassin supérieur de l'Alibori au Bénin, Journal of Applied Biosciences 108, 10543-10552, 2016.

Boko, M. : Climat et communautés rurales du Bénin : Rythmes climatiques et rythme de développement, Thèse d'Etat ès lettres, Dijon, France, 607 pp., 1988.

Claude, J. : Rapport de synthès, in : Les Hommes face aux sécheresses : Nordeste brésilien, Sahel africain [en ligne], Paris : Éditions de l'IHEAL, https://doi.org/10.4000/books.iheal.1216, 1989

Dardel, C. : Entre désertification et reverdissement du Sahel : Diagnostic des observations spatiales et in situ, Thèse de Doctorat, Université Paul Sabatier, Toulouse III, France, 200 pp., 2014.

Fonds international de développement agricole (FIDA) : Gestion des ressources naturelles et de l'environnement : Moyens d'existence résilients pour une utilisation durable des biens naturels, Rome (Italie), 52 pp., 2011.

Gillet, P., Vermeulen, C., Feintrenie, L., Dessard, H., et Garcia, C. : «Quelles sont les causes de la déforestation dans le bassin du Congo ? Synthèse bibliographique et études de cas », BASE [En ligne], 20, 183-194, 2016.

Hosonuma, N. et al. : An assessment of deforestation and forest degradation drivers in developing countries, Environ. Res. Lett., 7, 044009, 2012.

Ndiaye, A. et Ndiaye, P. : Changement climatique, dégradation environnementale et quête d'utilisation des ressources naturelles. Occasional Paper No. 21, The African Capacity Building Foundation (ACBF), Hararé (Zimbabwé), 50 pp., 2013.

OCDE : Ressources naturelles et croissance pro-pauvres : Enjeux économiques et politiques, Paris (France), CEDEX 16, 189 pp., 2009.

OMM et GWP : Manuel des indicateurs et indices de sécheresse, Genève, Suisse, 52 pp., 2016. 
Sanou, K., Amadou, S., Adjègan, K., et Tsatsu, K. D. : Perceptions et stratégies d'adaptation des producteurs agricoles aux changements climatiques au nord-ouest de la région des savanes du Togo, Agronomie Africaine, 30, 87-97, 2018.

Toko Imorou, I., Arouna, O. Zakari, S., Djaouga, M., et Thomas, O. : Évaluation de la déforestation et de la dégradation des forêts dans les aires protégées et terroirs villageois du bassin cotonnier du Bénin. Conférence OSFACO : Des images satellites pour la gestion durable des territoires en Afrique, Cotonou, Bénin, hal02189556, 2019.

Wicander, S., Helfgott, A., Bailey, M., Munroe, R., Ampomah, G., Diouf, A., Devisscher, T., et Corrigan, C. : Planification de la résilience et de l'adaptation pour les communautés des aires protégées, Guide par étapes. Cambridge : UNEP-WCMC, 112 pp., 2016.
Yabi, I. : Paysannat vivrier face aux incertitudes pluviométriques de la seconde saison agricole dans la commune de Didja au SudBenin, Revue de Géographie de l'Université de Ouagadougou, 3, 145-164, 2019.

Yabi, I. : Sécheresse pluviométrique dans la zone soudanienne au Bénin : Manifestations et Implications pour une agriculture climato-résiliente, Dynamiques Spatiales et Développement : Revue semestrielle du Laboratoire d'Etudes des Dynamiques Urbaines et Régionales, 2, 115-134, 2018.

Yabi, I. et Afouda, F. Extreme rainfall years in Benin (West Africa), Quaternary International, 262, 39-43, https://doi.org/10.1016/j.quaint.2010.12.010, 2012. 